\title{
Sobre las perspectivas pedagógicas para la educación virtual en Colombia
}

\author{
Karolina González Guerrero* \\ José Eduardo Padilla Beltrán** \\ Diego Armando Rincón Caballero*
}

Artículo recibido: 15-05-2011 y aprobado: 04-05-2012

\section{About educational prospects for virtual education in Colombia}

Abstract: This article is aimed at

Resumen: El presente artículo busca otorgar importancia a la revisión histórica, contextual y prospectiva de la educación virtual en Colombia y su interacción con el uso de las tecnologías de la información y la comunicación, teniendo en cuenta la descripción y puntualización de elementos teóricos, en aras de analizar su incursión en la educación superior. Esto se consigue a través de una revisión exhaustiva de diversas fuentes, textos y citas, en las cuales se puede construir un marco de antecedentes e investigaciones acerca de los aspectos generales de la transición del proceso virtual-presencial en la estructuración de diversos modelos alternos como el $b$-learning. A raíz de la interpretación, se llega a establecer límites, términos y condiciones para su percepción en instituciones de educación superior, desde un sistema de causalidad que permita llevar a cabo una retroalimentación para mejorar los procesos de formación en dichos espacios.

Palabras clave: Educación superior, Educación virtual, implementación de las TIC, modelo b-learning, modelos de formación.
Keywords: Virtual education, implementation of TIC, $b$-learning model, higher education models.

1 Articulo de reflexión resultado del proyecto de investigación:"Evaluación del docente en contextos b-learning en educación superior" Cód. EDU 743 - financiado por la Universidad Militar Nueva Granada, (2010-2011).

* Doctorando en educación y multimedia - Universidad de Aveiro (Portugal). Docente investigadora - Universidad Militar Nueva Granada. Grupo de Investigación PYDES- Pedagogía y Didáctica en la Educación Superior.rolina.gonzalez@unimilitar.edu.co, kgonzalezg@gmail.com

** Doctor en Educación - Newport University USA. Director Departamento de Educación - Universidad Militar Nueva Granada. Investigador Grupo de Investigación PYDES Pedagogía y Didáctica en la Educación Superior. eduardo.padilla@unimilitar.edu.co,edopadilla@gmail.com

** Licenciado en Diseño Tecnológico, Universidad Pedagógica Nacional. Joven Investigador Grupo PYDES Pedagogía y Didáctica en la Educación Superior. Universidad Militar Nueva Granada.

diego.rincon@unimilitar.edu.co,dccaballero@gmail.com 


\section{Introducción}

El siglo XXI impone nuevas oportunidades y grandes desafíos debido a las innovaciones de las tecnologías de la información y comunicación [TIC]. En este sentido, el ámbito educativo está experimentando importantes cambios como consecuencia de la inclusión tecnológica; los tradicionales paradigmas de enseñanza y aprendizaje están siendo modificados por la integración de TIC en el currículo. En plena era de la sociedad del conocimiento, la distribución del poder depende de quién maneja y apropia la información (Carvalho, 2006; Pablos, 2007).

Por lo tanto, la educación superior y muy enfáticamente las universidades, han entendido este nuevo escenario de profundos cambios y han comenzado a ajustar sus proyectos curriculares, dirigidos a estudiantes con diversas necesidades y variados estilos y ritmos de aprendizaje (Henao y Zapata, 2002). Es así como se han emprendido progresos y/o grandes proyectos tecnológicos, cuyo objetivo se centra en analizar la actitud y visión de los docentes hacia nuevas perspectivas pedagógicas para la enseñanza y aprendizaje, en las cuales la interacción y el trabajo colaborativo pasan a ser factores relevantes para la articulación de proyectos educativos soportados a través de plataformas virtuales.

Según las cifras que ofrece la reciente medición de las tecnologías de información y comunicación realizadas por el Departamento Nacional de Estadística, en el sector de la educación superior, muestra que prácticamente la mayoría de las universidades colombianas cuentan con campus virtual y herramientas de apoyo basadas en Internet. Este hecho ha posibilitado el surgimiento de una progresiva oferta educativa en la modalidad a distancia (e-learning) y últimamente en la modalidad combinada o b-learning (presencial/virtual).

La inclusión de medios y tecnologías, especialmente en la educación superior, representa retos y cambios en las estrategias de enseñanza, en cuanto al quehacer pedagógico que asume el docente (De Miguel, 2006; Pablos, 2007). Actualmente, existe una fuerte apuesta por los contextos $b$-learning entendidos como una combinación de actividades presenciales y las herramientas virtuales de la educación a distancia, en los que se potencia lo mejor de cada uno de los contextos mencionados (Bonk y Graham, 2006). Entonces, el b-learning se presenta como un contexto en el cual las transiciones pedagógicas y los modelos de formación, han dado cabida a integrar diversas perspectivas. En ese orden de ideas, el artículo desglosa diversos estudios al respecto, para describir estos cambios pedagógicos.

\section{La educación virtual en Colombia}

\section{Una breve mirada a la historia de la educación virtual en Colombia}

A nivel de formación superior, la educación virtual en Colombia tiene sus inicios en los años ochenta del siglo pasado (Facundo, 2002). Ha sido promovida por medio de modalidades de educación a distancia, teniendo un crecimiento paulatino con respecto a otras instituciones que comenzaron a implementar la educación virtual durante el siglo XX con propiedad (Valzacchi y Asinsten, 2004). En 1982, como lo afirma Facundo (2002), se crea la Universidad 
del Sur, que posteriormente se conocería como la UNAD, dando prioridad y financiación a instituciones que promulgaran este tipo de educación, con base en estudios y aportes trabajados desde la parte conceptual por la Unesco, entre los que se encuentra la creación de redes ALFA, consideradas como comunidades educativas, conformadas por países europeos y algunos latinoamericanos (Restrepo, 2006).

En contraste a lo descrito anteriormente, se hace mención al incremento de la educación superior en América Latina, hablando de una oferta considerable; Colombia se sitúa detrás de Brasil y México en cuanto a la inclusión de nuevos programas de posgrado (Zapata, 2010). También se menciona como parte de la revisión histórica que se hace en cuanto a la inmersión de la educación virtual en el ámbito de la educación superior, los aportes del Instituto Internacional para la Educación Superior en América Latina -IIESALC-, organismo que hace un análisis de la conceptualización de la educación virtual partiendo del uso de las tecnologías de la información y la comunicación [TIC], al recalcar dos aspectos fundamentales (Facundo, 2002):

1. Términos y limitantes de la educación que el estudio en mención ha preferido citar como educación a distancia-virtual.

2. La diferencia que hay con respecto a la educación virtual y lo referente a informática educativa.

Debido a las características de desarrollo y las demandas educativas sobre las que el país se mueve en cuestiones de formación superior, las universidades e instituciones han mostrado su interés por fomentar la educación virtual bajo nuevos modelos pedagógicos que estén mediados por los recursos de tecnologías para la comunicación y la información (Facundo, 2002), buscando llevar a cabo la interactividad y la consecución sistemática del saber con apoyo en redes como Internet. El uso de Internet como medio posibilitador de aprendizaje, deja entrever el déficit existente en Latinoamérica frente al uso de estas herramientas virtuales con respecto a otros continentes. A continuación se presenta un consolidado general sobre la adaptación de Internet a inicios del siglo XXI (Zapata, 2010):

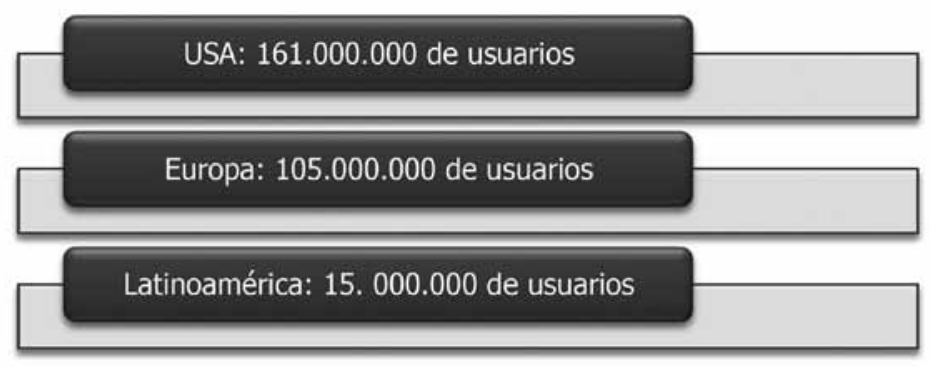

Figura No. 1: Usuarios con conexión a Internet (2002), desde la perspectiva de las TIC 
La figura No. 1, deja ver que apenas el 3.5\% de personas tenía una conexión a Internet en el 2002 (Facundo, 2002). Esto, visto desde la perspectiva sobre el uso de las TIC como medio educativo para la formación, denota la falta de recursos de orden económico y técnico que permitan una mayor oferta educativa a nivel virtual, en la cual la ruptura de paradigmas comienza a debatir y abatir las barreras de espacio-tiempo, más aún al consolidar medios para la accesibilidad informativa (Valzacchi y Asinsten, 2004) en donde los roles, medios y ambientes de aprendizaje constituyen nuevas miradas hacia lo que Zapata (2010) expone como una gran esperanza para la ampliación de la oferta educativa en espacios de formación superior.

\section{Las TIC en el contexto educativo}

Es preciso, entonces, a la par de mostrar un plano histórico sobre la educación virtual en nuestro país, verificar de forma contextual los elementos que constituyen y fomentan los procesos de teleformación apoyados en las herramientas TIC. Con ánimo de explorar más a fondo sobre los avances que ha tenido la educación virtual en la actualidad, es importante mostrar un plano comparativo, como lo resalta Henao (1992), sobre elementos característicos de la enseñanza de tipo virtual con respecto a lo que ofrece la educación tradicional, sin llegar a demeritar ninguna y, por ende, entrar en juicios de valor hacia la incidencia de cada ámbito en el plano pedagógico y educativo.

En primera instancia, se hace necesario destacar las diferencias que muestra la educación basada en las TIC con respecto a la tradicional: lo concerniente a la modificación de espacios, roles y métodos característicos de la educación virtual frente al paradigma tradicional o presencial, de acuerdo a los medios y manejos que se le den por parte de la institución, y su interrelación con paradigmas contemporáneos, entre ellos la era digital y la globalización como epicentro político, social y económico (Henao, 1992). Reforzando esta comparación de medios, se puede hacer un énfasis más detallado en el aspecto de divergencia en la mirada tradicional y la virtual de los roles y las interacciones que pueden coexistir con base en las dinámicas presentadas por cada tipo de formación:
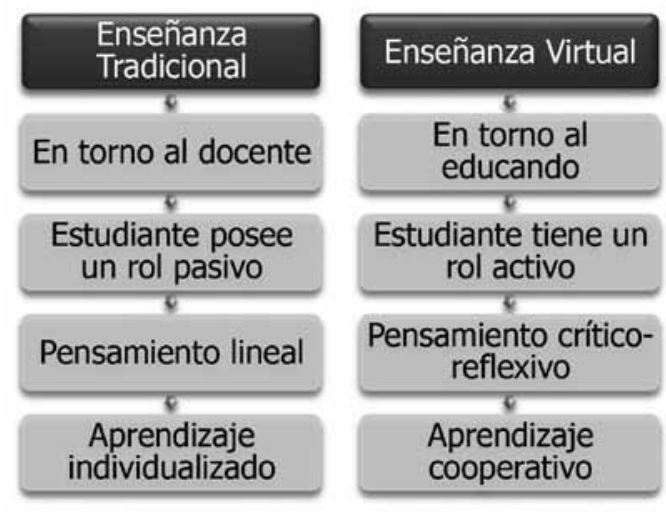

Figura No 2: Roles e interacciones en la enseñanza tradicional y virtual

De la figura No. 2, cabe la pena resaltar que no se trata de categorizar o ponderar la relevancia o algún tipo de favorabilidad en alguna de las dos enseñanzas. En lo que se quiere hacer énfasis es en lo que aclara Henao (1992), con respecto al deber correspondiente del docente de apropiarse de estas nuevas tecnologías y dar espacio a nuevos enfoques pedagógicos, que le permitan convertirse en un propiciador de aprendizaje centrado en la pertinencia contex- 
tual del ser como agente social y cultural introducido en la actual era globalizada.

Una manera de hacer cierta asociación entre ambos elementos, tanto el tradicional y el virtual, está en lo que Guarneros, Silva y Pérez (2009) denominan sistemas educativos mixtos y en línea, haciendo alusión al concepto de combinación como un desarrollo de modelo educativo que permite tomar los aspectos favorables de la educación presencial y los elementos que identifican la educación a distancia. Aún hoy en día, se presentan una serie de polémicas y de controversias con respecto al uso de las TIC y los espacios virtuales para el desempeño educativo (Barbera y Badia, 2005). No obstante, dentro de este marco contextual cabe añadir que este modelo educativo basado en la relación de elementos presenciales y virtuales, en la contemporaneidad se da como una alternativa que pretende alcanzar un desarrollo competitivo demarcado por solventar las limitantes de oferta académica y suplir, por medio de estrategias y procesos de aprendizaje dinámico, una formación de calidad para incentivar un desarrollo profesional e "integral" en el educando (Aguado y Arranz, 2005); es un aspecto latente en la comunidad educativa, donde cada vez más los elementos de la informática y la teleformación incrementan la gestión y la creación de nuevas comunidades científicas para el prospecto de nuevos saberes y generación de conocimiento, en torno a la innovación y articulación de paradigmas pedagógicos (Padilla, Páez y Montoya, 2008).

En Colombia, el uso de las TIC crece a un ritmo acelerado. En gran medida lo demuestran Calle y Molina (2009) con el impacto del uso de Internet para el 2009 en América Latina, con un $31.9 \%$ de incidencia en diversas regiones geográficas. La educación virtual fundamentada en las conexiones y redes, establece en el año 2009, por medio del congreso colombiano, una reforma presentada a modo de Ley TIC (Calle y Molina, 2009) como epicentro legal que denota una orientación hacia la generalización en el uso apropiado de dichas tecnologías en pro de la comunicación, la educación y el progreso orientado a la calidad social. Sin embargo, la notoria diferencia frente a experiencias de implementación y mejoramiento de la educación virtual a nivel de otros países latinoamericanos, norteamericanos y europeos, radica en la falta de estándares y lineamientos educativos y políticos que trasciendan a las instituciones de educación superior (IES). Por consiguiente, la virtualidad aún carece de reformas y mejoras determinadas a promulgar la conciencia del buen uso y la importancia de la implementación de planes metaevaluativos como parte de los factores de calidad en las IES.

\section{Prospectiva de las TIC orientadas a la educación superior}

Estos sistemas educativos basados en experiencias compartidas, en las cuales se presentan sesiones de modo presencial y espacios de interacción virtual, mejor conocidos como b-learning, expresión inglesa que significa proceso formativo combinado (Quitián, 2009). Se empieza a consolidar un componente de formación en línea, sopesado en una herramienta e-learning y un modelo instructivo para desarrollar un sistema de competencias caracterizadas por el programa o énfasis que se desea alcanzar (Aguado y Arranz, 2005). Sumado a ello, está el impacto que hay en el uso de las 
TIC como elemento de agilización en los procesos comunicativos e informativos dentro del ámbito de la educación superior (Méndez, Rivas y Del Toro, 2007). Entonces, estos medios de interacción bidireccionales promulgados por el b-learning, permiten hacer más ágil el desarrollo comunicativo, logrando que la interacción entre docente y estudiantes $\mathrm{y}$, a su vez, entre los mismos educandos, sea mucho más enriquecedora por las connotaciones colaborativas (Barbera y Badia, 2005) que puedan haber para alcanzar las metas dentro de la formación, específicamente hablando de la educación superior.

De una manera prospectiva y como lo expone Vera (2008), en el siglo XXI la marcha acelerada de las nuevas herramientas y servicios que ofrecen las TIC, conlleva a asumir grandes retos en muchos planos, no solo de manera profesional y formativa: además, se están rompiendo esquemas y paradigmas que a la larga modifican los núcleos sociales, en especial si se refiere al ámbito de la educación, en la cual el acceso informático cada vez es más avasallante, esto reflejado en nuevos medios y estrategias que recurren a nuevas alternativas didácticas, reforzadas por tecnologías que dan flexibilidad en relación a elementos como tiempo y espacio.

Este auge contemporáneo de las TIC nace como esperanza en la forma de afrontar los retos pedagógicos y educativos en el presente (Mayorga y Madrid, 2010). Quizá a modo de actualización, o en una sentencia más rigurosa de acuerdo a lo descrito por Vera (2008), quien posee y maneja mejor la información es una persona que obtiene un poder inimaginable. Méndez, Rivas y Del Toro (2007), a partir de los estudios realizados por Hoffman y Novak, plantean un análisis de los agentes que intervienen en la comunicación, distinguiendo principalmente la sincronía temporal, la interacción bidireccional y el tipo de formato de locución como los aspectos que pueden verse afectados o revolucionados con la inmersión en las TIC, en tanto epicentro de transformación social y en la manera de acceder al conocimiento hoy en día.

La Unesco y el Instituto Internacional para la Educación Superior en América Latina (IIESALC), demuestran a través de los estudios de Facundo (2002) el auge de las TIC, transformado en experiencias exitosas por las universidades latinoamericanas, en las que aumenta el número de programas virtuales o $b$ learning. Países como Argentina con la universidad de Quilmes, Mar del Plata, Rosario y otras tantas, reportan un alza del $42 \%$ de las ofertas de programas que convergen en modalidades virtuales; Colombia con universidades como la Católica del norte, Universidad Abierta y a Distancia (UNAD), EAFIT, Universidad Nacional, entre otras, demuestran un aumento del $40 \%$, teniendo mayor acogida en programas de pregrado; Brasil, por medio de instituciones tales como Sao Paulo, Brasilia, Campinas, SENAC, Prohecto Bra. Interlegis, entre otras, consolidan un aumento similar al de Argentina; Luego países como México con la Universidad de Monterrey y Chile con la UNIACC y la Universidad Mayor, establecen un aumento del 22\% y $75 \%$ respectivamente. 
A nivel norteamericano, Canadá le ha apostado a la educación virtual, presentando altos niveles de calidad debido al apoyo gubernamental que recibe esta modalidad formativa. Gutiérrez (2004) establece una proporción de instituciones con programas mixtos o virtuales de cuatro en razón de cinco instituciones a nivel de muestra en los Estados Unidos. El autor recalca que las políticas de calidad en Estados Unidos son semejantes a las canadienses, no obstante las diferencias radican en el replanteamiento pedagógico y la infraestructura utilizada para llevar a cabo los programas educativos a nivel superior.

Europa, por su parte, en países como España, muestra en universidades como la de Salamanca y Valencia, un gran adelanto en la oferta de programas virtuales, a partir de una infraestructura y objetos virtuales provistos desde diversas líneas de investigación encaminadas al estudio de la educación virtual. Así pues, España, Portugal y otros países, se perfilan para liderar el Sistema Educativo Europeo para la Educación Superior (EEES) (Turpo, 2010). A nivel de la Unión Europea, se trata de consolidar proyectos como el DELTA y el Fourth Frame Project (Gutiérrez, 2004), destinados a buscar lineamientos generales o estándares a implementar en la educación virtual o de naturaleza combinada (presencial y virtual).

\section{Análisis de inclusión de las TIC Procesos de teleformación apoyados en el uso de TIC}

Analizando a profundidad la inclusión de las TIC en el plano educativo, y más en el ámbito de la educación superior, se puede llegar a un concepto o categoría un poco confusa o, como lo declara Herrera (2006), algo ambigua. Por tal razón es importante enunciar que en ese proceso avasallante, la conceptualización para el plano educativo de orden superior está muy relacionada con la posibilidad de ofrecer y ampliar la oferta educativa para eliminar barreras en fronteras de comunidades y universidades (Vera, 2008). Así pues, el educando es el centro de estudio en el modelo formativo y a su vez se vuelve epicentro para la transformación de espacios virtuales que den nuevas reformas, pensadas en el mejoramiento de la calidad y los recursos educativos, con el principal objetivo de fortalecer los procesos comunicativos descritos por Barbera y Badia (2005) como interacciones telemáticas que favorecen, en cierta medida, la conformación de las comunidades virtuales de aprendizaje.

A continuación se presenta, en la figura No. 3, una gráfica que tiene como fin mostrar los aspectos interventores en el desarrollo teleformativo, teniendo en cuenta su aplicación en ámbitos de educación superior: 


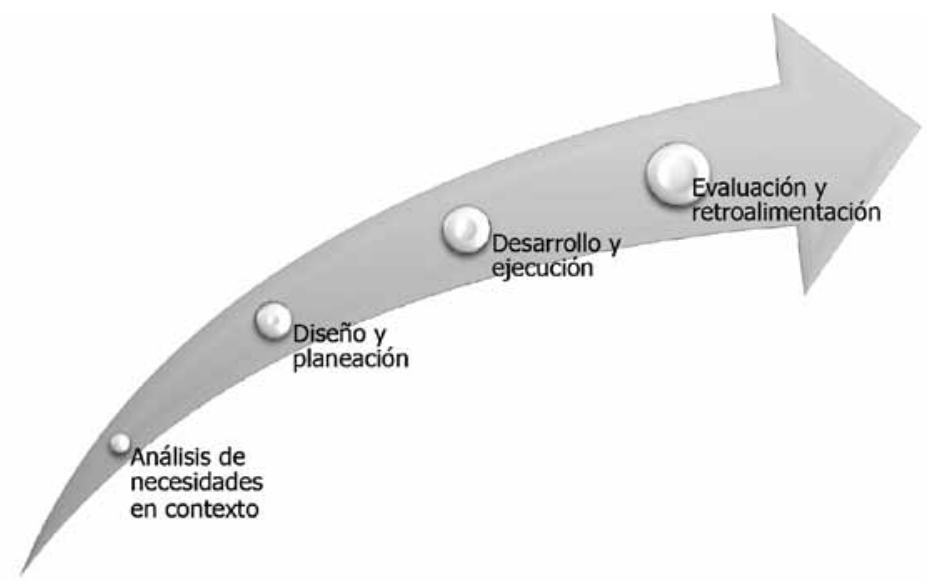

Figura No. 3: Aspectos que intervienen para el desarrollo a través de teleformación (Marcelo, 2005)

Cada uno de los temas resaltados en la anterior figura está precedido por el éxito de las fases anteriores, y de forma cíclica vuelven a plantearse para dar unanimidad en cuanto al ambiente, ya sea virtual o mixto de aprendizaje, a implementar. Aunque no hay que dejar de lado el hecho del componente flexivo (Marcelo, Puente, Ballesteros y Palazón, 2000), pues es muy importante en la relación de los procesos adaptativos y las necesidades que demarca el usuario o colectivo sobre el recurso virtual, para llegar a establecer su aprendizaje y formación bajo criterios de calidad.

Desde el punto de vista de la educación mixta o b-learning, la conceptualización de las TIC abocadas en el plano de la educación superior obtiene una realidad que resulta ser algo incipiente, y aunque, como lo recalca Marcelo et al. (2000), no es un factor de descontextualización o eminente atraso, las experiencias más relevantes e importantes se perfilan desde ciertas prácticas desarrolladas en educación continua o universidades especializadas en el tema (Vera, 2008). Desde una mirada más hacia los pensamientos de los profesores con respecto al uso de las TIC, un estudio realizado por López (2009) en el 2007, deja a la luz un resultado que manifiesta que el 64\% de los docentes utiliza este medio para la realización de actividades de formación, en tanto un $47 \%$ las aplica en acciones personales. De lo anterior, la deducción se centra en el creciente uso de las TIC; sin embargo, la reflexión gira en torno a si el uso es el adecuado y si es pertinente bajo el establecimiento de nuevos escenarios, partícipes para la formación y el uso de las nuevas tecnologías para fortalecer el proceso pedagógico y educativo (Marcelo et al., 2000).

\section{Modelos para la articulación de las TIC en el contexto de la educación superior}

Aunque en anteriores apartados se han mencionado algunos modelos procedentes de la deducción y conceptualización de las TIC en el marco de la educación superior, como el modelo flexible, modelos emergentes como el $b$-learning y los aspectos que refieren a una didáctica dentro de estos espacios 
virtuales, se hace necesario mencionar estos y otros elementos que fundamentan el uso de las TIC en espacios virtuales y mixtos, desde diversos recursos conceptuales y teorías que sustentan su utilización en la educación superior.

Un primer modelo para la estructuración conceptual y organizacional de las TIC es analizado a partir de los estudios de Cebrián (2005), quien muestra las siguientes categorías a partir de la relación e interacción tanto de los actores como de los ambientes partícipes en el campo de la educación superior:

Modelo transmitivo: según el estudio, está sopesado por las acciones del docente y en los medios y recursos informáticos que integra para dar a conocer de forma adecuada los desarrollos conceptuales incorporados en el entorno virtual o mixto.

Modelo interactivo: viene mediado por las relaciones del estudiante con el grupo y los docentes dentro del marco conceptual, social y formativo que ofrece el programa educacional, bien sea mixto o virtual.

Modelo colaborativo: refiere, dentro de lo que analiza el estudio, a la inclusión de las TIC como recurso que propicia un conocimiento basado en el desarrollo de estrategias cooperativas, permitiendo el crecimiento académico por medio del trabajo en equipo y el aprendizaje mediado por la colaboración para el desarrollo personal y profesional.

Otro modelo que puede ser citado, refiere en este caso al estudio de Vera (2008), el cual centra la categorización de su estudio en la inmersión de las TIC dentro de la educación, a partir de los elementos que se transforman o pre- sentan cambios, en contraste a lo que se había venido enunciando como plano comparativo de la educación tradicional y la educación virtual. Así pues, a continuación se comentan dichos factores que engloban el modelo descrito:

- Modelo pedagógico: hace referencia al cambio de paradigma, en cuanto a la importancia de centrar esfuerzos en el aprendizaje basado en el estudio y la aplicación de las teorías ofrecidas desde los estilos y ritmos de aprendizaje en un orden cognitivo, y las relaciones propiciadas por las TIC para llevar a cabo un aprendizaje cooperativo.

- Transfronterización de la oferta educativa: un proceso que habla de cobertura y llevar a más personas y comunidades la educación como medio de inclusión cultural y desarrollo profesional, hablando de la educación superior.

- Gestión institucional: una vinculación y estructuración más precisa que permita establecer mejores procesos comunicativos en todas las dependencias y organismos aunados en la institución formadora.

- Estrategias de investigación: por medio de nuevas estrategias y acciones comunicativas, los procesos de socialización entre investigadores y grupos académicos se vuelven más efectivos y eficaces.

En aspectos más deductivos, se puede concebir una categorización a nivel de modelos influyentes dentro de las TIC en 
la educación superior, bajo tres aspectos fundamentales que guardan relación directa con un modelo más amplio, como lo es el b-learning y otros modelos, como aclara Vera (2008), subyacentes a los planteamientos y ofrecimientos de la educación mixta. En ese orden de ideas, los modelos presentados a continuación pueden verse vinculados con el b-learning a través de otros submodelos que actúan como nodos conectores en cuanto a las relaciones y estrategias que fortalecen la participación activa entre docentes, estudiantes y gestores inmersos en el proceso de formación bajo el aprovechamiento de las TIC:
El primer modelo educativo a tener en cuenta es el de orden flexible (De Benito y Salinas, 2005), considerado bajo una relación con el concepto que ofrecen Mayorga y Madrid (2010) de modelo como representación preconcebida y de orden reflexivo dentro del espacio educativo relacionado en el marco pedagógico. Esto lleva a considerar si el componente flexible debe ser condescendiente con las características y las necesidades de los actores en el proceso formativo. Los componentes flexibles, como lo recalcan De Benito y Salinas (2005), pueden ser

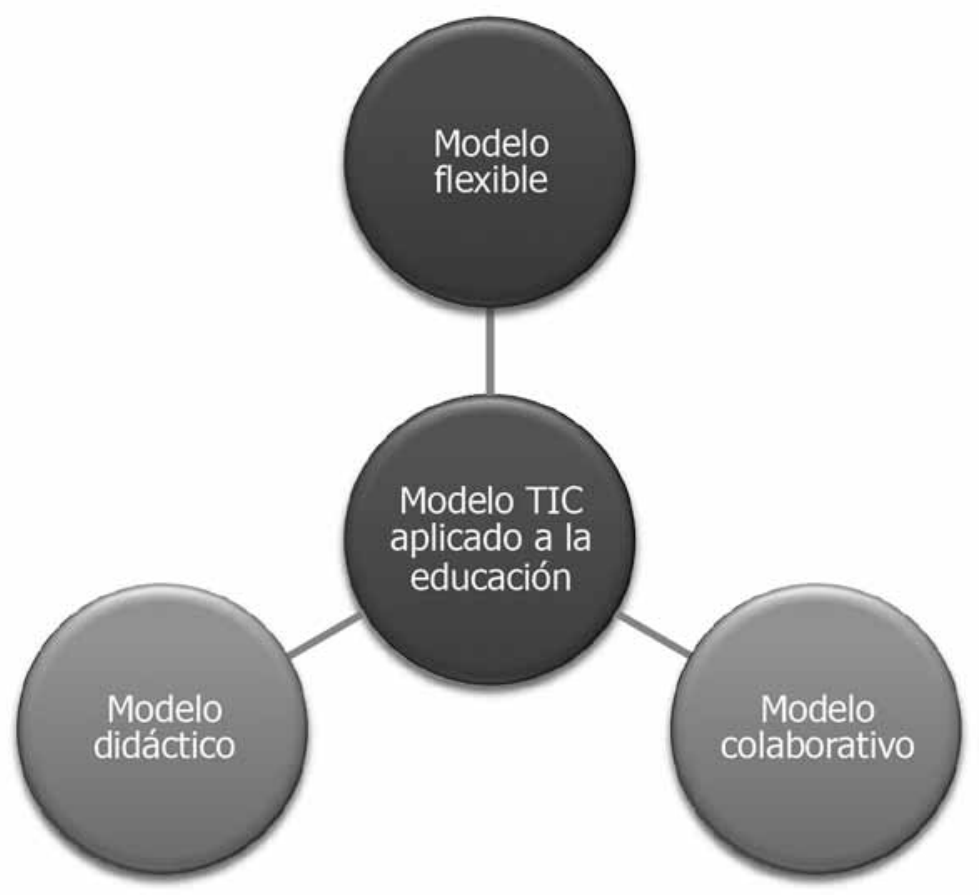

Figura No. 4: Modelos pedagógicos relacionados en el proceso de formación mediado por TIC 
adaptativos y por ende son subsecuentes a la estimulación de la inteligencia, como lo asevera Herrera (2006), basado en los estudios de Piaget con respecto a la modificación de estructuras mentales y aspectos cognitivos a partir de la presentación de variados esquemas, técnicas y medios por parte del formador (Mayorga y Madrid, 2010), para establecer lazos comunicativos-académicos y actitudinales dentro del proceso educativo.
En segunda instancia, se presenta el modelo didáctico, el cual es una manera de entender y justificar la dimensión de la práctica educativa (Mayorga y Madrid, 2010). Este modelo muestra caracterizaciones que presentan un plano comparativo en cuanto a los cambios que ha tenido la incursión pedagógica en la acentuación didáctica, de acuerdo a las transformaciones en medios y finalidades formativas:

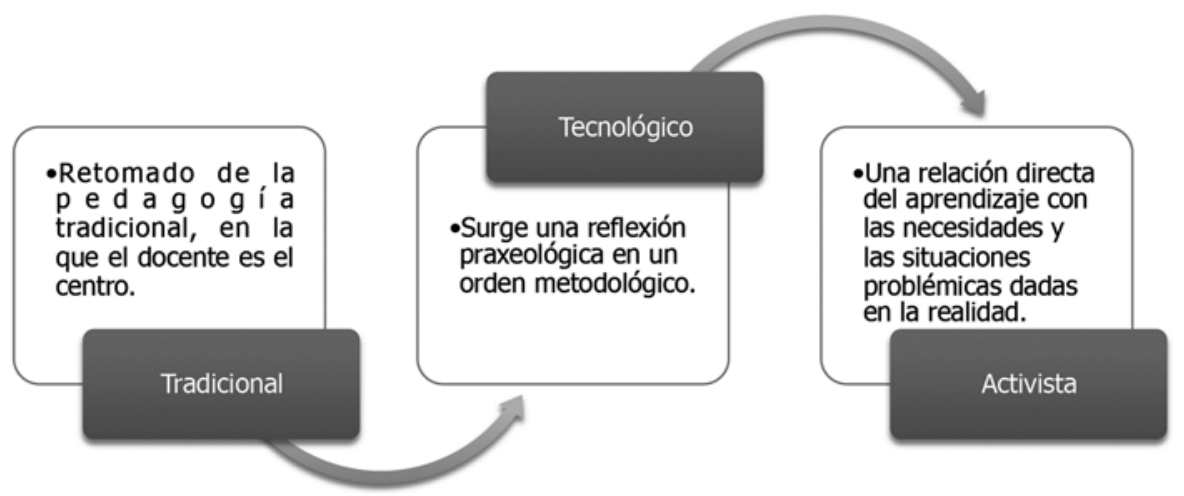

Figura No. 5: Transición del modelo pedagógico didáctico (Mayorga y Madrid, 2010)

El esquema de la figura No. 5 expone de forma progresista cada aspecto, debido a la relevancia que ha tenido y la incursión dentro del contexto pedagógico; claro está, ninguno es predominante y, por el contrario, cada uno ofrece diversos postulados que hoy en día se reflejan en la educación, más exactamente en espacios diversificados como aulas físicas o virtuales (Vera, 2008). Tampoco se puede olvidar que el modelo didáctico refiere al proceso de interpretar los aspectos relacionados con los productos teórico-prácticos abstraídos de la concepción de realidad, y por ende a la consolidación de teorías y paradigmas
(Mayorga y Madrid, 2010). Bajo esta generalidad, el modelo didáctico aplicado al uso de las TIC, más exactamente en espacios mixtos de aprendizaje, permite diversificar aún más el componente flexible, estudiando diversas perspectivas y medios que propicien nuevas alternativas para el proceso pedagógico en dichos espacios, bien sea virtuales esencialmente, o combinados como lo ofrece el b-learning.

Un tercer aspecto a considerar se fundamenta en la teoría de Vygotski, la cual será empleada a posteriori para determinar el margen teórico que demarca los aspectos pedagógicos y las 
teorías en los ambientes de aprendizaje de orden virtual o mixto. Sin embargo, en este subtema se pretende relacionar el entorno social como factor determinante en el proceso de aprendizaje (Herrera, 2006) con el modelo cooperativo, donde el trabajo en equipo, precedido de unos objetivos y metas, está mediado para facilitar y aprender recíprocamente de forma activa, bajo el desarrollo de actividades o contenidos mostrados en el proceso de formación. La riqueza de este modelo reside en tres aspectos fundamentales enunciados por Barbera y Badia (2005): la definición de objetivos dirigidos al grupo, una asignación de funciones específicas a cada integrante del grupo y la disposición de recursos para afrontar los retos y actividades, son aspectos fundamentales que consolidan el éxito del trabajo colaborativo.

Podemos asociar el modelo cooperativo bajo otros submodelos expuestos por Vera (2008) (ver figura No. 6), para determinar la incidencia del aprendizaje en diferentes ritmos y bajo apoyo grupal:

\section{Características para la vinculación de las TIC en la educación virtual a nivel superior}

Es indispensable, además de formular modelos que sugieran la participación de las TIC en escenarios de educación virtual o mixta de orden superior, entender cuáles son las características y los aspectos que giran en torno al uso de las TIC, con base en los modelos descritos anteriormente y en especial hacia la aplicación en los contextos de educación mixto b-learning. Quiere decir esto que describir los aspectos fundamentales llevará a idealizar los ambientes de aprendizaje propicios, dentro de los cuales se pueden mencionar dos aspectos fundamentales que identifican los espacios de interacción bien sea, como menciona Herrera (2006), de orden presencial, virtual o mixto, en tanto son de preceptos constitutivos y conceptuales (Marcelo y Puente, 2002). No obstante, se ha de recalcar el hecho de la existencia de distintos estudios que dan diversas miradas en cuanto a la caracterización
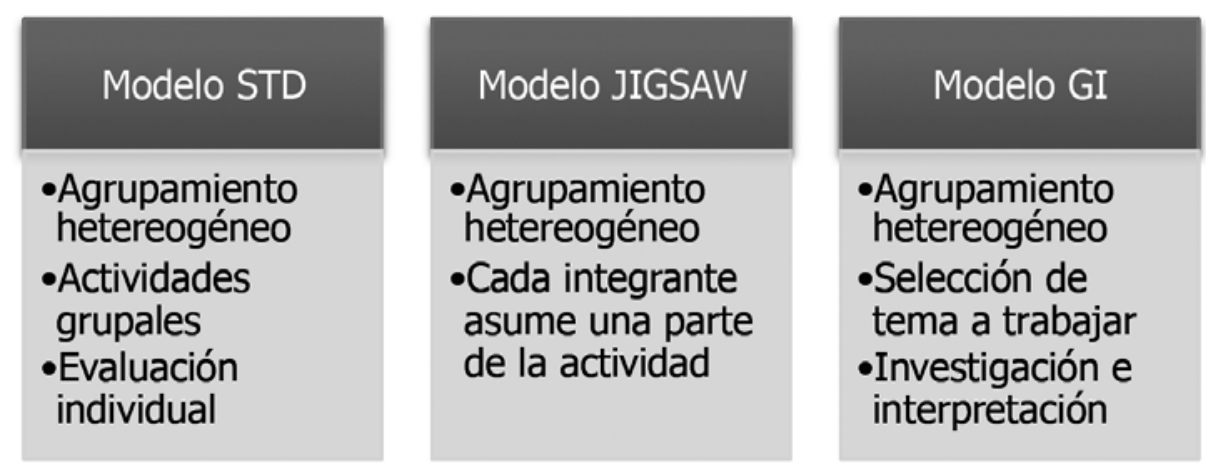

Figura No. 6: Submodelos expuestos por Vera (2008) para el modelo cooperativo 
para espacios de aprendizaje (Díez, Pérez y Sánchez, 2009). En cierta medida, algunas estarán relacionadas con los medios y plataformas que incurren en procesos de interacción de modo virtual: tal es el caso de Moodle y la importancia de la consolidación de entornos virtuales de aprendizaje (EVA) (López, 2009), o el desglose de las ventajas y las ideas primordiales que incentivan el uso de las TIC en contextos educativos (Quitián, 2009).

Según Marqués (2000) la educación, y en especial la formación ofrecida por espacios universitarios, estará confinada en desarrollos que puedan darse en el marco presencial y las generadas por medios virtuales a distancia, destacando el hecho de una personalización del aprendizaje bajo requerimientos y expectativas que posea el educando. Por otro lado, Marqués (2000) resalta la importancia de un cambio en cuanto a la relación de asistencia y nivel de producción por créditos acumulados, en vez de tener en cuenta un enfoque centrado en las habilidades y competencias (desde el pensamiento empresarial) que puede generar el estudiante desde sus propias necesidades.

En esa mirada empresarial, resulta pertinente describir que en la concepción de entornos virtuales o mixtos de aprendizaje, se opera un cambio de las capacidades de adaptación en forma organizada bajo procesos pedagógicos, elementos didácticos y tecnológicos (Marqués, 2000), y así consolidar un vínculo con los medios laborales desde los aciertos y falencias (López, 2009) que pueda tener el sistema educativo, en proyección hacia el modelo subsecuente para la estructuración de ambientes desde la orientación del educando (Quitián, 2009).

Para concluir este tema se hace un plano comparativo entre diversas características que ofrecen tres autores en particular, las cuales se ponen a consideración por tratar de establecer los elementos que deben caracterizar un ambiente de aprendizaje:

\begin{tabular}{|l|l|l|l|}
\hline \multicolumn{1}{|c|}{$\begin{array}{c}\text { Características (Mondéjar y } \\
\text { Vargas, 2007) }\end{array}$} & $\begin{array}{c}\text { Características (Herradón et } \\
\text { al., 2009) }\end{array}$ & Características (Herrera, 2006) \\
\hline Pedagógico & $\begin{array}{l}\text { Permite un aprendizaje } \\
\text { más dinámico }\end{array}$ & \\
\hline Psicológico & $\begin{array}{l}\text { Ritmo de aprendizaje } \\
\text { individualizado }\end{array}$ & $\begin{array}{l}\text { Colaboración, } \\
\text { motivación e implicación }\end{array}$ & Relaciones psicológicas \\
\hline Organizacional & $\begin{array}{l}\text { Es una fuente de reducción } \\
\text { de costes }\end{array}$ & Factores físicos \\
\hline Didáctico & $\begin{array}{l}\text { Posibilita un espacio } \\
\text { virtual de reunión para la } \\
\text { realización de trabajos }\end{array}$ & $\begin{array}{l}\text { Definición de problemas } \\
\text { bien enfocados }\end{array}$ & $\begin{array}{l}\text { Los recursos y medios } \\
\text { de interacción }\end{array}$ \\
\hline
\end{tabular}

Tabla No. 1: Comparación entre las características de diferentes modelos 
En lo concerniente a la perspectiva pedagógica, retomando cada una la incidencia de los modelos propuestos a nivel norteamericano, Estados Unidos presenta reformas curriculares que según Restrepo (2006), se solventan con un aprendizaje exploratorio del educando a partir de la tríada de tutoría, metacognición y flujos informativos orientados desde las herramientas proporcionadas por las aulas virtuales. Europa consolida un prospecto pedagógico orientado al humanismo y la conformación de interculturalidad a través de las comunidades académicas, es decir, la movilidad y el intercambio hacen parte de los componentes para desentrañar un aprendizaje colaborativo, propulsado por la interacción y las herramientas sincrónicas para una tutoría personalizada y mancomunada por educandos, expertos invitados y tutores del programa.

América Latina propende por una orientación pedagógica basada en la intersección competitiva, cuyo resultado sigue imperando más allá del proceso. Sin embargo, los confines sociales se establecen de manera reflexiva en el trabajo pedagógico constructivista, en el marco del aprendizaje por problemas y proyectos que puedan tener impacto sobre problemáticas en el entorno circundante (Amenta, 2006). La mezcla de un modelo tradicional y tecnológico, converge en muchos casos en una didáctica carente de interacción y sosegada en el campo investigativo, aunque algunos países como Chile, Argentina, Brasil y México han empezado a mostrar avances en este aspecto pedagógico, con una mayor flexibilidad desde el conectivismo hacia el construccionismo y el cognitivismo en sus programas de formación en las IES.

\section{Resultados y discusión}

En el presente artículo se trata de precisar desde una revisión de diversas referencias bibliográficas, el impacto que posee la formación virtual y, con especial atención, su articulación con medios presenciales en el ámbito de la educación superior. Así pues, en este proceso sistemático los resultados se enfocan en dar pertinencia y establecer una reflexión desde la importancia histórica, contextual y prospectiva acerca del impacto y uso de las TIC en la educación virtual en Colombia. Para presentar los resultados del ejercicio investigativo, se muestra, en primera instancia, una disertación pedagógica sobre las orientaciones pedagógicas que priman a la hora de fundamentar y proponer una educación virtual o $b$-learning (virtual y presencial), dando a conocer la relevancia de una mirada conceptual en los procesos de teleformación. Posteriormente, se usa un recurso de pregunta orientadora, la cual tendrá su consideración desde los temas expuestos en el documento, para dar continuidad a trabajos ulteriores que trasciendan en una investigación encaminada a revisar bajo estudio de casos o diversas IES, el impacto actual de las TIC y la educación virtual en la formación superior en Colombia.

\section{Teorías pedagógicas relacionadas con la educación virtual y el b-learning}

Ante el cambio paradigmático y pedagógico provisto por el impacto de las TIC en el sector educativo, se hace relevante analizar las acciones formativas que asume el docente y el estudiante, los tiempos de interacción y los espacios académicos que deberán ser en parte de manera presencial y en parte de manera 
virtual (Henao, 1992). Esto tiene, en cierto punto, respuesta desde la concepción de un sustento pedagógico-teórico que determine la orientación de la formación; por lo tanto, dentro de las teorías más influyentes y sobre las cuales recae en gran parte el sustento teórico del aprendizaje virtual y b-learning, están las siguientes (Vera, 2008):

- Conductismo: reflejada por la estimulación y la retroalimentación que se presenta en desarrollo de actividades y en la asistencia tutorial dada en modos virtuales.

- Constructivismo: a través de los desarrollos cooperativos e individuales que se dan al momento de indagar y construir conocimiento por medio de casos particulares y ejercicios de exploración.

- Cognitivismo: con respecto a estrategias y medios por los cuales se aprende de forma causal e indagando los fenómenos circundantes.

- Humanismo: por medio de la motivación ejercida por el tutor en medios virtuales y la observación subjetiva del grupo en formación desde los medios presenciales. Así como también la incidencia de la denotación de estilos y ritmos de aprendizaje.

La teoría constructivista es una de las más representativas e influyentes desarrolladas en los medios educativos en general (Quitián, 2009), obteniendo un tratamiento de dimensiones dirigidas a la solución de problemas (Díez, Pérez y Sánchez, 2009) por parte del estudiante, a través de procesos de inferencia y análisis pertinentes en el uso de las TIC por medios presenciales. En ese sentido, si el estudiante es el constructor activo de su propio saber, deberá ser consecuente con los siguientes postulados tratados por Díez et al. (2009):

1. La importancia de la interacción en el estudiante: el debate y el planteamiento de posturas a través de las dinámicas grupales, permite al educando generar nuevos esquemas de pensamiento, formando de manera crítica puntos de vista sobre determinado tema.

2. La problemática como factor cognitivo: la articulación de contenidos, objetivos y metas está ligada, en gran proporción, a los desarrollos conceptuales que se puedan manifestar por medio de problemas o necesidades que se presenten en el contexto del estudiante.

3. La facilidad de aprendizaje por medios colaborativos: en los espacios presenciales, y aún más en los ambientes virtuales, las herramientas que conlleven a interacciones sociales y estímulos de trabajo en equipo son propicias para determinar formas de pensamiento dirigidas a dar diversas miradas a una situación compleja.

Desde el enfoque humanístico presentado anteriormente también puede hacerse extensivo el estudio, al profundizar en cierta medida hacia los estilos o ritmos de aprendizaje; teoría que también es muy influyente en el análisis teórico de los espacios de formación 
centrados en el blended learning y, por otro lado, bajo las consideraciones de un proceso de autoaprendizaje (Henao, 1992) provistos por el propio educando al tomar responsabilidad actitudinal y de aptitud frente a su proceso formativo.

Estos constructos teóricos referentes a modelos y desarrollos pedagógicos son, en parte, fundamentales para determinar la importancia de concebir un desarrollo pedagógico que medie la inserción de las nuevas tecnologías en el ámbito de la educación superior. En esa instancia, como exponen Valzacchi y Asinsten (2004), los paradigmas cambiantes de las acciones del docente y el estudiante, en cuanto al cambio que se les ha otorgado con el impacto de dichas tecnologías, no ha sido del todo revolucionario, pues como los mismos autores aclaran, son adaptaciones teóricas que han venido trabajándose desde los postulados de Vigotsky y Piaget en el sentido constructivista, y por el lado cognitivista, desde los supuestos de Harvard, en especial el trabajo hecho por David Perkins en su estudio de la escuela inteligente (Valzacchi y Asinsten, 2004). Este último está centrado en una educación con miras a la comprensión como objeto primordial y reto que asume la educación contemporánea; de forma analógica, este hecho es el que persigue, también, la integración de nuevos espacios virtuales e innovadoras tecnologías aplicadas a la comunicación y la información en la educación, más exactamente en el orden superior. Es decir, como recalcan Valzacchi y Asinsten (2004): lo innovador solo adquiere sentido y pertinencia con relación prospectiva al crecimiento global en comparación a otras épocas.
Lo anterior hace hincapié a determinar, específicamente, la manera de actuar de las IES frente al avance de la educación virtual y mixta, consagrando una renovación pedagógica que más allá de combinar diversos enfoques pedagógicos, determine una posición frente al contexto y las necesidades particulares de la sociedad, en este caso Colombia y las particularidades sociales, culturales, políticas y económicas que impactan ineludiblemente a la acción educativa del país. A continuación, se plantean dos preguntas orientadoras que buscan finalizar este apartado, con un nivel propositivo acerca de la importancia de la formación virtual y mixta en Colombia y por qué debería ser un tema relevante para ser investigado en el marco del impacto de las TIC en el contexto universitario.

\section{¿Por qué un contexto de formación mixto en nuestro país?}

Desde las perspectivas referentes a historia, contexto y prospectiva, el análisis de la formación mixta en la educación superior vista desde el panorama nacional, ha tenido un gran impacto desde su incentivación en los años ochenta del siglo anterior (Facundo, 2002). En ese sentido, la justificación de su incursión en el país hace alusión a dos aspectos importantes; uno concerniente a las trasformaciones culturales, sociales y pedagógicas hacia las cuales se hizo mención en el documento desde las investigaciones ejercida por la Unesco y autores como Zapata (2010); por otro lado, desde la oferta y demanda que ofrece la educación superior en Colombia, donde el b-learning surge como alternativa para cubrir de forma demográfica, entre otros aspectos sociales y 
económicos, llegando de forma asincrónica y rompiendo esquemas de espacios físicos, sin dejar de lado la riqueza que muestra el modelo mixto combinando las fortalezas de ambos medios (Henao, 1992).

En esa medida, la importancia de los modelos virtuales y mixtos de aprendizaje en el país, se refleja en el acelerado uso de las TIC que se ha manifestado en el marco educativo (Mayorga y Madrid, 2010), llegando a estimar su impacto en novedosas formas de establecer núcleos colaborativos y aportes mediáticos centrados en las consideraciones sociales en formación de orden superior. Por ende, llevar a cabo una vinculación con debates y aportes hechos de manera presencial (Méndez et al., 2007).

\section{¿Cuál es la incidencia de las TIC en los procesos de teleformación en el marco de la educación superior?}

Con la revisión bibliográfica se llegó a la caracterización de diversos modelos, que permiten establecer un marco comparativo a la luz de diversos autores sobre diferentes estrategias, concepciones pedagógicas y de orden didáctico (Cebrián, 2005), supeditadas a bases conceptuales que permiten orientar la estructuración de un modelo general al cual se apela en el escrito como b-learning (Vera, 2008). No obstante, como resultado particular en este tema, se refleja que a partir de la oferta educativa y la eventual vinculación institucional a este macromodelo, se llega a diversas miradas y la aplicación de modelos que a la par pueden ser asociados con otros y entenderse como híbridos nacidos por la concepción flexible que estos medios formativos ofrecen (De Benito y Salinas, 2005).
Esto confrontado con la conceptualización de procesos teleformativos dada desde la percepción de Marcelo (2005), lleva a procesos reflexivos en estudio de casos que permitan dar cuenta de la incidencia de las TIC en procesos a través de teleformación desde la caracterización docente, pues como muestra el estudio de revisión, hay en promedio $64 \%$ de docentes de educación superior que utilizan estos recursos tecnológicos para generar actividades formativas (López, 2009). Es decir que en gran parte las TIC son acogidas por los educadores; su uso y aplicación estarán mediados por modelos y estrategias, las cuales deben ser analizadas a profundidad para dar cuenta de su pertinencia social, pedagógica y contextual.

\section{Conclusiones}

Durante la estructuración del artículo y bajo los datos estadísticos ofrecidos en los diversos estudios analizados, se pudo contrastar el crecimiento paulatino que ha mostrado el uso de las TIC en nuestro país, en comparación con el ascenso del uso de las TIC para la educación en Norteamérica y países europeos. Adicionalmente, los estudios focales sobre el tema son los más importantes, sin demeritar los adelantados a nivel colombiano que han hecho organizaciones como IIESALC y otros organismos en diferentes países latinoamericanos.

En el proceso contextual del análisis de las TIC y su impacto a nivel nacional, se encuentra, a modo comparativo, que los sistemas de educación se han transformado desde los cambios en roles, ambientes y medios por los cuales se fundamentan las TIC en la educación. En ese sentido, la enseñanza virtual 
determina nuevas orientaciones pedagógicas que irrumpen en esquemas de participación más asociados a dinámicas activas de pensamiento divergente, prevaleciendo el papel del estudiante como agente de transformación académica en esa cosmovisión antropológica y cultural de entender lo virtual como un escenario con magnitud de estructura social.

Muchos autores plantean un posible fracaso del e-learning con respecto a su uso en el ámbito educativo, en lo cual surgen como opción nuevos modelos que convergen en combinaciones de esquemas presenciales y a distancia, en donde el paso de las TIC en la formación superior consolide un apoyo a la demanda educativa que se presenta actualmente en el país, minimizando recursos y atendiendo a las políticas y estándares de orden nacional e internacional, las cuales en el plano actual, se centran en las competencias y la calidad desde la concepción metafórica de la aldea global.

Respecto a los modelos presentados para caracterizar el impacto de las TIC en el contexto de las IES, los procesos de teleformación no solamente afectan de manera paulatina el quehacer pedagógico, en la misma transformación paradigmática de la teleformación: la oferta educativa, la interculturalidad y las gestiones institucionales comprometen una acción transformadora que permita abordar las dimensiones sociales, humanísticas y tecnológicas desarrolladas a nivel global, todo en pro de articular procesos de calidad para el mejoramiento de la educación con base en el crecimiento de la demanda en el sector profesional.

Según los hallazgos encontrados en la literatura, los modelos tradicionales, construccionistas y cognitivos adquieren mayor relevancia para establecer un diseño pedagógico que se articule con el ambiente virtual de formación. No obstante, debido al cambio paradigmático de las acciones pedagógicas a realizar por el docente y el educando, cobra mayor ímpetu el carácter humanístico, toda vez se presente un escenario formativo dotado de una calidad para motivar, comunicar e interactuar con los estudiantes de una manera dialógica, crítica y reflexiva ante el uso de las TIC y la importancia que tienen en la actual era de la información. Consecuentemente, las IES deberán comprometer una visión curricular y didáctica sobre estrategias para el buen uso de las herramientas y procesos estructurados en ambientes virtuales de aprendizaje, así como las ventajas y desventajas provenientes de estos medios de formación en la flexibilidad y grado de autonomía, no solamente delegada a los educandos, sino a los diversos participantes que integran el equipo de trabajo en el programa educativo. 


\section{Referencias}

Aguado, D. y Arranz, V. (2005). Desarrollo de competencias mediante blended learning: un análisis descriptivo. $R e$ vista de Medios y Educación, 26, 79-88.

Amenta, G. (2006). La educación virtual en ciencias sociales en Latinoamérica. En CNA: Entornos virtuales en la educación superior. (pp. 89-99). Bogotá: Corcas Editores.

Barbera, E. y Badia, A. (2005). Hacia el aula virtual: actividades y aprendizaje en la red. Revista Iberoamericana de Educación, 36 (9).

Bonk, C \& Graham, C. (2006).The handbook of blended learning global perspectives, Local Designs (ed.), Jay Cross (Foreword), Publisher: John Wiley \& Sons, Inc. Pfeiffer, p 624.

Calle, C. y Molina, T. (2009). Diseño, desarrollo e implementación de un diplomado en línea en gestión y soporte de equipos médicos. CES Medicina, 23 (2), 73-80.

Carvalho, C. (org.) (2006). E-learning e formação avançada. Casos de sucesso no Ensino Superior da Europa e América Latina. Porto: Edições Politema.

Cebrián, M. (2005). Los procesos de innovación didáctica en el marco del espacio europeo de educación superior. España: Universidad de Salamanca.

Díez, R., Pérez, C. y Sánchez, A. (2009). Experiencias y metodologías "b-learning” para la formación y evaluación en competencias genéricas en Ingeniería. Revista: La Cuestión Universitaria, 5. pp. 33-45.

De Benito, B. y Salinas, J. (2005). Situaciones didácticas en los entornos virtuales de enseñanza aprendizaje (EVEA) en la enseñanza superior: elaboración de un instrumento de análisis. Grupo de Tecnología Educativa. Congreso internacional EDUTEC 2005.

De Miguel, M. (Coord.) (2006). Metodologías de enseñanza y aprendizaje para el desarrollo de competencias. Orientaciones para el profesorado universitario ante el espacio europeo de educación superior. Madrid: Alianza Editorial.

Facundo, A. (2002). La educación superior a distancia/virtual en Colombia. Recuperado el 22 de Marzo de 2011, de:

http://portales.puj.edu.co/didactica/ PDF/Tecnologia/EducacionvirtualenColombia.pdf

Guarneros, E., Silva, A. y Pérez, C. (2009). La innovación educativa y tecnológica en la educación superior de México, una empresa pendiente. Cuadernos de Educación y Desarrollo, 1 (2).

Gutiérrez, J. A. (2004). Definición de un modelo pedagógico para la educación virtual en el CES (1st ed.). Medellín: Editorial CES.

Henao, O. (1992). El aula escolar del futuro. Revista Educación y Pedagogía, 4 (8-9), 87-96.

Henao, O. y Zapata, D. (2002). La enseñanza virtual en la educación superior (1a Ed.). Colombia: Icfes. pp. 85.

Herradón, R., Blanco, J., Pérez, A. y Sánchez, J.A. (2009). Experiencias y metodologías "b-learning" para la formación y evaluación en competencias genéricas en Ingeniería. La Cuestión Universitaria, 5, 33-45.

Herrera, M. (2006). Consideraciones para el diseño didáctico de ambientes virtuales de aprendizaje: una propuesta basada en las funciones cognitivas del aprendizaje. Revista Iberoamericana de Educación, 38 (5),1-19. 
López, M. (2009). Formación de los docentes y la promoción de innovaciones educativas. Una experiencia B-learning en el curso -La educación bolivariana-. Revista Iberoamericana de Educación, 49 (1).

Marcelo, C. (2005). Evaluación en e-learning: Compartiendo algunas experiencias. España: Universidad de Sevilla.

Marcelo, C. y Puente, D. (2002). Aprender con otros en la red. Investigando las evidencias. Virtual Educa, p. 6.

Marcelo, C., Puentes, D., Ballesteros, M. y Palazón, A. (2000). Diseño, desarrollo y evolución de la formación a través de Internet. Barcelona: Gestión.

Marqués, P. (2000). Impacto de las TIC en la enseñanza universitaria. España: UAB.

Mayorga, J. y Madrid, D. (2010). Modelos didácticos y estrategias de enseñanza en el espacio europeo de educación superior.

Méndez, A., Rivas, A. y Del Toro, M. (2007). Entornos virtuales de enseñanza aprendizaje. Las Tunas: Ministerio de Educación Superior Editorial Universitaria. Centro Universitario de Las Tunas.

Monjejar, J.A. y Vargas, J. (2007). Docencia virtual en universidades presenciales: experiencia en la Universidad de Castilla-La Mancha. RIED, 10 (2), 207-228.

Pablos, J. (2007). The methodologic change in the European Higher Education area \& the role played by the information and communication technologies, Universidad de Sevilla (España) Revista de Educación a Distancia, 10 (2),15-44.
Padilla, J., Páez, C. y Montoya, D. (2008). Creencias de los docentes acerca del uso de las tecnologías de información y comunicación. Revista Educación y Desarrollo Social, vol.2 (2).

Quitián, S. (2009). Relaciones entre modelos de lectura: focalizada y panorámica y estilos cognitivos dependiente e independiente de campo, en ambientes de aprendizaje b-learning. Bogotá: Universidad Pedagógica Nacional; Facultad de Ciencia y Tecnología.

Restrepo, B. (2006). Tendencias actuales en la educación superior: rumbos del mundo y rumbos del país. Revista Educación y Pedagogía. , 18 (46), 79-90. Medellín: Universidad de Antioquia,

Turpo, O. (2010). Contexto y desarrollo de la modalidad educativa blended learning en el sistema universitario iberoamericano. Revista Mexicana de Investigación Educativa, 15 (45), 345-370.

Valzacchi, J. \& Asinsten, J. (2004). Implicancias del procesamiento didáctico en los entornos virtuales de aprendizaje. Argentina: Buenos Aires.

Vera, F. (2008). La modalidad blendedlearning en la educación superior. Chile: Rancagua.

Zapata, M. (2010). Evaluación de competencias en entornos virtuales de aprendizaje y docencia universitaria. Revista de Educación a Distancia, Docencia Universitaria en la Sociedad del Conocimiento, 1. 